\title{
Contribución al conocimiento de la flora vascular de la provincia de Zamora (España)
}

\author{
Patricio Bariego Hernández y María Santos Vicente ${ }^{2}$
}

Resumen: Bariego, P. \& Santos Vicente, M. 2011. Contribución al conocimiento de la flora vascular de la provincia de Zamora (España). Bot. Complut. 35: 59-64.

Se presentan y comentan 24 taxones de interés corológico para la provincia de Zamora (España), 7 de los cuales son novedad provincial: Allium ericetorum, Arctostaphylos uva-ursi, Armeria duriaei, Arum cylindraceum, Carex humilis, Cerinthe major subsp. gymnandra y Doronicum plantagineum. Todos los taxones han sido poco citados en el territorio de estudio y algunos además poseen un notable interés biogeográfico. Se comentan además algunas citas previas a tenor de las revisiones taxonómicas más recientes.

Palabras clave: corología, flora vascular, nuevas citas, Zamora, España.

Abstract: Bariego, P. \& Santos Vicente, M. 2011. Contribution to the knowledge of the vascular flora of the province of Zamora (Spain). Bot. Complut. 35: 59-64.

24 taxa of chorological interest of the province of Zamora (Spain) are remarked and commented, of which 7 are cited here for the first time: Allium ericetorum, Arctostaphylos uva-ursi, Armeria duriaei, Arum cylindraceum, Carex humilis, Cerinthe major subsp. gymnandra and Doronicum plantagineum. All taxa are poorly referenced in the studied area and several of them show a remarkable biogeographic interest. Some previous records are commented in the light of the latest taxonomic reviews.

Key words: chorology, vascular flora, Zamora, Spain.

\section{INTRODUCCION}

A lo largo de los últimos años se ha venido realizando un intenso trabajo de prospección de la flora vascular zamorana que ha tenido como resultado la publicación de diversas notas florísticas (Bariego \& Gutiérrez García 2003, Bariego \& Rico 2003, Bariego et al. 2004, Bariego \& Gastón 2005, Bariego \& Santos Vicente 2005, entre otros). En la presente nota se recogen varios taxones que no habían sido citado en la provincia (se señalan con ${ }^{*}$ ) y se aportan nuevos datos corológicos para otros taxones cuya presencia en el territorio es escasamente conocida.

Para cada taxón se indican los datos referentes a su localización geográfica (municipio, toponimia, UTM $1 \times 1$ $\mathrm{km}$ y altitud), el hábitat donde ha sido observado, la fecha y autor/es de la recolección y un número de identificación del pliego personal de P. Bariego (PB) y/o la referencia al herbario de la Universidad de Salamanca (SALA) donde se han depositado los pliegos. Además se comentan brevemente algunos datos referidos a su distribución geográfica e interés biogeográfico, con especial atención a las localidades más cercanas que se conocen.

\section{RESULTADOS}

Aconitum napellus subsp. lusitanicum Rouy

ESPAÑA: ZAMORA: Alcañices, Molino la Raya, ribera del Angueira, 29TQG2117, 735 m, aliseda, 19-VIII-1999, P. Bariego s.n., SALA 102697.

Nueva población zamorana de este geófito ligado a bosques ribereños que ya era conocido en la ribera del río Aliste (Valle 1984: 296, sub Aconitum napellus subsp. neomontanum (Wulfen) Gáyer) y en el tramo portugués del río Angueira (Rozeira 1944: 100).

${ }^{1}$ C/ Candelaria Ruiz del Árbol, E-47018 Zamora, España.pbariego@hotmail.es

${ }^{2}$ Departamento de Botánica, Universidad de Salamanca, Avda. Licenciado Méndez Nieto s/n, E-37007 Salamanca, España.msantos@usal.es Recibido: 13 enero 2011. Aceptado: 20 enero 2011 
Adonis microcarpa DC.

ESPAÑA: Zamora: Madridanos, Bamba, Teso del Viso, 30TTL8392, $730 \mathrm{~m}$, cuestas margosas básicas, herbazales sub-nitrófilos, 17-IV-2003, P. Bariego PB 3224.

Añadimos una nueva localidad, no muy alejada de las únicas conocidas en la provincia (Giráldez 1987: 108), de esta ranúnculacea rara en la provincia.

\section{*Allium ericetorum Thore}

ESPAÑA: Zamora: Muelas de los Caballeros, arroyo del Ferradal, 29TQG2074, $1180 \mathrm{~m}$, zona higroturbosa, 10-09-1991, B. Casaseca \& E. Rico s.n., SALA56629; LEÓN: Castrocontrigo, Torneros de la Valdería, entre los arroyos Cabañas y Cefreo, 29TQG2374, 1100m, 4-09-1990, B. Casaseca, X. Girádez \& E. Rico s.n., SALA56631.

Con motivo de la revisión corológica para Flora iberica se han puesto a la luz estas recolecciones, tanto zamoranas como leonesas, en zonas de turbera en el extremo oriental de la Sierra de la Cabrera. Se trata de un taxón de distribución restringida al norte de la Península Ibérica que no había sido citado previamente en los trabajos florísticos que estudian esta zona, ni en el ámbito de Zamora (García Río \& Navarro 1994) donde es novedad provincial, ni en el de León (Nieto Feliner 1985).

\section{Anemone nemorosa $\mathrm{L}$.}

ESPAÑA: Zamora: Justel, Monte Velilla, arroyo de Fuente del Buey, 29TQG1874, 1120 m, aliseda, 20-VIII-2001, P. Bariego \& A. Gallego PB 60; Espadañedo, Vega del Castillo, Sierra de la Cabrera, Tigeo, 29TQG1874, $1150 \mathrm{~m}$, aliseda, 12-V-2002, P. Bariego \& A. Galle$g_{0}$ PB 702.

Muy localizada en determinados enclaves del noroeste de la provincia, añadimos dos nuevas localidades en la vertiente zamorana de la Sierra de la Cabrera, ampliando su área provincial (Aedo et al. 1993: 355).

\section{*Arctostaphylos uva-ursi (L.) Spreng.}

ESPAÑa: Zamora: Trabazos, Latedo, 29TQG0519, $640 \mathrm{~m}$, pinar negral, 20-VI-2004, P. Bariego PB 2627.

Aportamos una interesante localidad, que constituye la primera referencia en la provincia de esta ericácea rara en la mitad occidental de la depresión del Duero. Recientemente denunciábamos también su presencia en la provincia de Valladolid (Bariego \& Santos Vicente 2005: 73).

\section{*Armeria duriaei Boiss.}

ESPAÑA: Zamora: Rosinos de la Requejada, Escuredo, entre el Alto del Peñón y el Vizcodillo, 29TQG0375, 1920 m, matorrales bajos de Genista sanabriensis y Vaccinium myrtillus, 15-VIII-2001, $P$. Bariego PB 2050.

Novedad para la flora de la provincia de Zamora. Citada de las cumbres del Teleno (Nieto Feliner 1985: 72), aportamos ahora una localidad en la zona alta de la sierra de la Cabrera donde también viven otras especies del género (Armeria ciliata (Lange) Nieto Feliner y A. langei Boiss.). Atendiendo a los comentarios personales de Nieto Feliner, a quién agradecemos su ayuda en la correcta determinación, habría que descartar las citas previas de este taxón en la provincia, debidas probablemente a los problemas nomenclaturales del taxón.

\section{*Arum cylindraceum Gasp.}

ESPAÑA: ZaMoRa: Villarino de Manzanas, arroyo Cabrón, 29TQG0839, 640 m, zarzales, 20-V-2002, P. Bariego PB 1737; Trabazos, Nuez de Aliste, arroyo del Puerto, 29TQG0325, $560 \mathrm{~m}$, rebollar, 29-IV-2003, P. Bariego PB 3128.

Aportamos localidades concretas de este taxón, cuya única referencia provincial previa es la de Flora iberica (Galán \& Castroviejo 2007: 291). Según el mapa de distribución peninsular (Draper \& Roselló-Graell 1997: 315) la localidad más cercana es la portuguesa de Vimioso. A tenor del tratamiento propuesto en Flora iberica y de lo comentado por Draper \& Roselló-Graell (l.c.) habría que poner en duda las citas previas para el occidente zamorano de su congénere Arum maculatum $\mathrm{L}$.

\section{*Carex humilis Leyss.}

ESPAÑA: Zamora: Moral de Sayago, El Hoyón, 29TQG4100, $650 \mathrm{~m}$, pastizales silicícolas en claros de quejigar, 19-III-2002, P. Bariego PB 706.

Primera cita para la provincia de Zamora. Las referencias más cercanas que se conocen son las leonesas de Destriana (Llamas 1984: 163) y las vallisoletanas de Encinas de Esgueva (Fernández Alonso 1986: 525). Luceño \& Escudero (2008: 210) señalan que se trata de una especie de apetencias basófilas y con una distribución centrada en las montañas y parameras calizas del $\mathrm{N}$ y E peninsular. Podría considerarse un ejemplo más de las 
plantas calcícolas que alcanzan estos emplazamientos occidentales por el corredor del Duero (Bariego \& Santos Vicente 2005).

\section{*Cerinthe major subsp. gymnandra (Gasp.) Rouy}

ESPAÑA: Zamora: Fermoselle, Ambas Aguas, 29TQF1274, 460 $\mathrm{m}$, herbazales subnitrófilos en bancales abandonados, 15-IV-2002, $P$. Bariego \& A. Gallego PB 1473.

Novedad provincial; se conocían varias localidades en los Arribes del Duero, pero todas ellas en la provincia de Salamanca (Carrasco 1994).

\section{Chrysosplenium oppositifolium $\mathrm{L}$.}

ESPAÑA: Zamora: Pías, Villanueva de la Sierra, valle del Bibey, 29TPG6360, $1340 \mathrm{~m}$, abedular, 20-VIII-2002, P. Bariego PB 1883; Porto, valle del Bibey, 29TPG7673, $1400 \mathrm{~m}$, acebeda, 18-V-2003, $P$. Bariego PB 3323; Hermisende, pr. núcleo urbano, 29TPG7448, $850 \mathrm{~m}$, setos en bordes de prados, 07-VIII-2002, P. Bariego, A. Gallego \& M. Santos Vicente PB 1929.

Aportamos nuevas localidades para este taxón, restringido al extremo noroccidental de la provincia, cuya única referencia provincial previa es la de Casaseca et al. (1990: 116). En la Sanabria Alta puede llegar a ser localmente abundante, viviendo en enclaves rezumantes y zonas muy húmedas en el seno de bosques frescos y densos como abedulares 0 acebedas.

\section{*Doronicum plantagineum L.}

ESPAÑA: Zamora: Galende, San Martín de Castañeda, Cueva de San Martín, 29TPG8470, 1380 m, acebeda, 22-V-2002, P. Bariego PB 763; Trabazos, Nuez de Aliste, arroyo del Puerto, 29TQG0325, 560 $\mathrm{m}$, rebollar, 29-IV-2003, P. Bariego PB 3126.

Primera cita provincial para esta especie que crece en ambientes forestales de las comarcas de Sanabria y Aliste. Las localidades más próximas que conocemos son de la Maragatería en León (Llamas 1984).

\section{Echium rosulatum Lange subsp. rosulatum}

ESPAÑA: Zamora: Puebla de Sanabria, Ungilde, 29TPG9755, $965 \mathrm{~m}$, herbazales nitrófilos en cunetas húmedas, 06-VII-2004, P. Bariego $\mathrm{PB} 2949$.
Añadimos una nueva localidad a las señaladas por Giráldez \& Rico (1986:34) en el extremo noroccidental de la provincia.

\section{Euonymus europaeus L.}

ESPAÑA: Zamora: Cubo de Benavente, monte La Dehesa, 29TQG3366, $805 \mathrm{~m}$, arbustedo mixto en orlas de rebollar, 31-VII-1999, P. Bariego s.n. (SALA 101759); Riofrío de Aliste, Requejada, 29TQG3432, $760 \mathrm{~m}$, orlas arbustivas de aliseda, 12-IX-1999, P. Bariego s.n. (SALA 101758); Justel, Sierra de la Cabrera, Monte Velilla, 29TQG1875, $1380 \mathrm{~m}$, robledal sobre bloques de cuarcita, 20-VIII-2001, P. Bariego \& A. Gallego PB 75; Trabazos, Nuez de Aliste, arroyo del Puerto, 29TQG0325, 560 m, rebollar, 29-IV-2003, P. Bariego PB 3129.

Nuevas citas que completan su distribución en la provincia, donde sólo era conocido en las inmediaciones del Lago de Sanabria (Amich 1982: 214).

\section{Frankenia pulverulenta L.}

ESPAÑA: Zamora: Zamora, Las Chanas, arroyo del Zape, 30TTL6695, $645 \mathrm{~m}$, praderas-juncales halo-subnitrófilas en las inmediaciones del arroyo, 05-VII-2003, P. Bariego PB 2884.

Nueva localidad en el occidente provincial a añadir a las previamente conocidas en el centro-este (Ladero et al. 1984, García Río \& Navarro 1994: 71).Se amplía así su área de distribución a los interesantes afloramientos subsalinos situados al oeste de la capital zamorana, en la zona de transición entre la cuenca sedimentaria y la penillanura sayaguesa.

\section{Genista carpetana Lange}

ESPAÑA:ZAMORA: Galende, San Martín de Castañeda, pr. Laguna de Peces, 29TPG8771, $1700 \mathrm{~m}$, pastizales psicroxerófilos, 12-V-2002, P. Bariego \& A. Gallego PB 922; Galende, 29TPG9165, $1010 \mathrm{~m}$, vallicar con aulagas, 29-V-2002, P. Bariego PB 1020; Galende, Pedrazales, 29TPG9165, $1000 \mathrm{~m}$, matorrales de Calluna vulgaris, 20-VIII-1996, $P$. Bariego PB 287; Villardeciervos, Cional, pr. núcleo urbano, 29TQG2048, $860 \mathrm{~m}$, pastizales húmedos, 09-VIII-2003, P. Bariego PB 2864.

A pesar de que este endemismo ibero-norteafricano ha sido recurrentemente citado en las zonas elevadas de la Sierra Segundera (Sánchez Anta \& Navarro 1985: 318, García López et al. 1992: 63, Mayor et al. 1983: 57), aportamos ahora nuevas localidades, algunas a altitudes notablemente bajas dado que en Flora iberica (Talavera 2000) se señala su límite inferior en los $1.300 \mathrm{~m}$. Dos de estas 
localidades se emplazan en la comarca de Sanabria y una más meridional en las inmediaciones de la Sierra de la $\mathrm{Cu}$ lebra, donde no había sido citada previamente (Valle 1982).

\section{Hypericum hirsutum L.}

ESPAÑA: Zamora: Trabazos, Nuez de Aliste, arroyo del Puerto, 29TQG0425, $550 \mathrm{~m}$, pastos húmedos entre melojos, 9-VII-1991, E. Rico, X. Giráldez, M. J. Pérez-Hornero \& M. M. Martínez-Ortega s.n., SALA 108260.

Se trata de una planta rara en la provincia cuya única localidad provincial es la que se publica ahora. Este material sirvió en su momento de referencia para la indicación provincial de Flora iberica (Ramos Núñez 1993: 179).

\section{Myosurus sessilis S. Watson}

ESPAÑA: Zamora: Peñausende, Sexmil, 30TTL6278, 800 m, pastizales temporalmente encharcados, 01-V-2002, P. Bariego PB 1440.

Añadimos una nueva localidad provincial a la única conocida de Muga de Sayago (Montserrat 1967: 131, sub. Myosurus heldreichii Lev.). En la localidad que señalamos ahora, no muy alejada de la anterior, convive con otra ranúnculacea de interés, Ranunculus batrachioides subsp. brachypodus G. López

Narcissus minor subsp. asturiensis (Jord.) Barra \& G. López

ESPAÑA: Zamora: Mayalde, barranco de la Degollada, 30TTL6468, 900 m, pastizales húmedos, 11-IV-2003, P. Bariego PB 3274.

Amplíamos el área de distribución de este taxón aportando esta localidad en el extremo meridional provincial, que sumamos a las ya publicadas de la sierra de la Culebra (Bariego \& Gutiérrez García 2003: 52). Los trabajos en el marco de Flora iberica han servido para su reciente tipificación (Ajax asturiensis Jord.) (Aedo 2010: 134).

Paeonia officinalis subsp. microcarpa (Boiss. \& Reut.) Nyman

ESPAÑA: ZAMORA: Porto, Vega de Conde, 29TPG8475, $1610 \mathrm{~m}$, canchal, 19-VII-2002, P. Bariego PB 1216; Porto, entre Vega de Tera y el Moncalvo, 29TPG8272, $1640 \mathrm{~m}$, canchal, 20-VIII-2002, P. Bariego PB 2367; Trabazos, San Martín del Pedroso, 29TQG0321, 530 m, pizarras, manchas de encinar, 29-IV-2003, P. Bariego PB 3378; Trabazos, Nuez de Aliste, arroyo del Puerto, 29TQG0325, $560 \mathrm{~m}$, rebollar, 29-IV-2003, P. Bariego PB 3121; Figueruela de Arriba, Riomanzanas, La Cuadrona, 29TQG0539, 720 m, encinar, 03-VII-2004, P. Bariego PB 2539.

Nuevas localidades de esta llamativa planta que ya fue indicada para la comarca de Sanabria por Losa (1949: 77). Aportamos ahora nuevas localidades en el ámbito de la Sierra Segundera y otras más meridionales en la comarca de Aliste, donde no había sido citada previamente (Valle 1982).

\section{Petrorhagia saxifraga (L.) Link}

ESPAÑA: Zamora: Villar del Buey, Pinilla de Fermoselle, el Picón de la Riviese, 29TQF1982, 420 m, olivar, 15-XII-2001, P. Bariego PB 572.

Planta ligada a roquedos en ambientes termófilos que en la provincia se restringe a los cañones fluviales del Tormes y Duero. Previamente señalada en la confluencia de ambos ríos (Sánchez Rodríguez 1986: 372), aportamos aquí la segunda referencia provincial en una localidad cercana en los Arribes del Duero.

\section{Saxifraga lepismigena Planellas}

ESPAÑA: Zamora: Pías, Villanueva de la Sierra, valle del Bibey, 29TPG6360, $1340 \mathrm{~m}$, abedular, 20-VIII-2002, P. Bariego PB 1885; Porto, valle alto del Tera, base de Trevinca, 29TPG8378, $1640 \mathrm{~m}$, roquedos salpicados por resalte en bordes de arroyo, 19-VII-2002, P. Bariego PB 1067; Porto, Circo de Vidulante, 29TPG8479, 1880 m, megaforbios en base de roquedos rezumantes, 14-VIII-2002, P. Bariego \& Sánchez Agudo PB 2468; Porto, valle alto del Tera, 29TPG8378, 1680 m, turbera en arroyo naciente, 14-VIII-2002, P. Bariego \& Sánchez Agudo PB 2463; Trabazos, San Martín del Pedroso, 29TQG0321, 530 m, pizarras, manchas de encinar, 29-IV-2003, P. Bariego PB 3375.

Salvo la indicación provincial de Flora iberica (Vargas 1997: 170) no conocemos otras referencias para la provincia de Zamora. Las localidades que aquí aportamos, emplazadas en suelos rezumantes y roquedos umbrosos, pertenecen a la Sierra Segundera y la comarca de Aliste.

\section{Serratula nudicaulis (L.) DC.}

ESPAÑA: Zamora: Porto, Vega de Conde, 29TPG8475, $1610 \mathrm{~m}$, canchal, 19-VII-2002, P. Bariego PB 1215. 
Tan sólo conocida en ambientes forestales del extremo meridional provincial (Giráldez 1986: 32), añadimos ahora una localidad en las montañas sanabresas de la Sierra Segundera.

\section{Spiraea hypericifolia subsp. obovata (Waldst. \& Kit.) H. Huber}

ESPAÑA: Zamora: Moral de Sayago, Vuelta de la Figal, 29TQG4200, 580m, claros de encinar con quejigos, 31-VII-1999, P. Bariego s.n., SALA 101763; Cubo de Benavente, Monte La Dehesa, 29TQG3366, $805 \mathrm{~m}$., arbustedo mixto en orlas de rebollar, 31-VII-1999, P. Bariego s.n., SALA 101766; Almaraz de Duero, Los Infiernos, 30TTM6000, $620 \mathrm{~m}$, repisas y grietas en afloramientos de pizarras, 27IV-2002, P. Bariego PB 1535; Otero de Bodas, Val de Santa María, embalse de Agavanzal, 29TQG2950, $800 \mathrm{~m}$, afloramientos rocosos, 28VIII-2002, P. Bariego PB 1850; Alcañices, Vivinera, arroyo de Urrietalagua, 29TQG2216, $740 \mathrm{~m}$, jaral en claros de encinar, 09-IX2002, P. Bariego PB 601; Peque, Oterino, ribera del río Negro, 29TQG2560, $840 \mathrm{~m}$, afloramientos rocosos en claros de encinar, 13-VI2004, P. Bariego PB 2601; Figueruela de Arriba, Riomanzanas, La Cuadrona, 29TQG0539, 720 m, encinar, 03-VII-2004, P. Bariego PB 2540.

Nuevas localidades a añadir a la anteriormente publicada de la sierra de la Culebra (Valle 1984: 298). Con estas nuevas citas se amplía el área de distribución conocida para este arbusto disperso por el occidente provincial, desde los Arribes del Duero a la comarca de la Carballeda, donde no había sido citado previamente (Sánchez Rodríguez 1983, García Río \& Navarro 1994). Se cubre así el vacío provincial detectado en los mapas de distribución ibérica (Romo 1983, Sesma \& Loidi 1992) y confirmando la distribución para esta zona que indica Morales (1998: 9) en Flora iberica.

\section{Vaccinium uliginosum $\mathrm{L}$.}

ESPAÑA: Zamora: Porto, circo de la Surbia, 29TPG8178, 1860 $\mathrm{m}$, nacientes en roquedos rezumantes, 19-VII-2002, P. Bariego PB 1226; Porto, Peñas de Bulante, 29TPG8776, $1960 \mathrm{~m}$, roquedos umbrosos, 20-VI-2002, P. Bariego \& A. A. Gallego PB 1148; Porto, pr. Laguna de Patos, 29TPG8578, $1980 \mathrm{~m}$, brezal húmedo, 30-VII-2002, P. Bariego PB 846; Rosinos de la Requejada, Escuredo, sierra de la Cabrera, Vizcodillo, 29TQG0674, 1990 m, pastizales psicroxerófilos con Genista sanabriensis, 15-VIII-2001, P. Bariego PB 85.

La única referencia conocida para Zamora era la indicación provincial de Flora iberica (Villar 1993: 521). Aportamos varias localidades de este taxón en la Sierra Segundera (Porto) y una en la sierra de la Cabrera (Rosinos de la Requejada); respecto a esta última, había sido citado en la vertiente leonesa (Nieto Feliner 1985: 92) pero no en la zamorana (García Río \& Navarro, 1994).

\section{BIBLIOGRAFÍA}

AEDO, C. 2010. Typyfications of the names of Iberian accepted species of Narcissus L. (Amaryllidaceae). Acta Bot. Malac. 35. 133-142.

Aedo, C.; Aldasoro, J. J.; Argüelles, J. M.; Díaz Alonso, J. L.; González Del Valle, J. M.; HerRá, C.; Laínz, M.; Moreno Moral, G.; Patallo, J. \& SÁnchez Pedraja, 0. 1993. Contribuciones al conocimiento de la flora cantábrica. Fontqueria 36: 349-374.

Amich GarCíA, F. 1982. Algunas plantas del valle de Sanabria (Zamora). Anales Jard. Bot. Madrid 39(1): 214-215.

Bariego Hernández, P. \& Gutiérrez García, J. L. 2003. Notas corológicas de plantas vasculares en la sierra de la Culebra (Zamora, España). Stud. Bot. Univ. Salamanca 22: 49-53.

Bariego Hernández, P. \& Rico Hernández, E. 2003. Novedades florísticas para la Cuenca del Duero (España). Lazaroa 24: $129-130$.

Bariego HernándeZ, P. \& Santos Vicente, M. 2005. Algunas plantas de interés corológico en el extremo occidental de la cuenca sedimentaria del río Duero (Valladolid y Zamora). Fl. Montib. 29: 72-83.
Bariego Hernández, P.; Delgado Sánchez, L. \& Santos VICENTE, M. 2004. Aportaciones al conocimiento de la pteridoflora de la Cuenca del Duero (España). Bot. Complut. 28: 57-59.

BARIEgo, P. \& Gastón, A. 2005. Aproximación al catálogo de orquídeas silvestres de la provincia de Zamora. Ecología 19: 149-164.

CARrasco, M. A. 1994. Cerinthe gymnandra Gasparr. (Boraginaceae) on the Iberian Peninsula. Bot. Complut. 19: 75-82.

Casaseca, B.; Giráldez, G.; Guillén, A. \& Rico, E. 1990. Manifestaciones eurosiberianas en la sierra Segundera, Zamora (prolongación del eje Pirenaico-Cantábrico). Monogr. Inst. Piren. Ecol. Jaca 5: 111-120.

Draper, D. \& Roselló-Graell, A. 1997. Distribución de Arum cylindraceum Gasp. (Araceae) en la Península Ibérica. Anales Jard. Bot. Madrid 55(2): 313-319.

Fernández Alonso, J. L. 1986. Fragmenta chorologica occidentalia, 306-392. Anales Jard. Bot. Madrid 42(2): $517-525$

Galán, A. \& Castroviejo, S. 2007. Arum L. En: S. Castroviejo, M. Luceño, A. Galán, P. Jiménez Mejías, F. Cabezas \& 
L. Medina (Eds.), Flora iberica 18: 283-293. Real Jardín Botánico, CSIC, Madrid.

García López, P.; Lauzurica, P.; Rey Quiroga, P. \& Roa MeDINA, A. 1992. Informe botánico del parque natural del Lago de Sanabria y sus alrededores. Monografías de la REN. Junta de Castilla y León.

García Río, R. \& NaVARRo AndRés, F. 1994. Flora y vegetación cormofíticas de las comarcas zamoranas del Pan, Tera y Carballeda. Stud. Bot. Univ. Salamanca 12: 23-202.

Giráldez Fernández, X. \& Rico Hernández, E. 1986. Algunas plantas de Zamora. Lazaroa 9: 131-138.

GiRÁLDEZ FeRnÁNDEZ, X. 1986. Contribución al conocimiento de la flora zamorana. II. Lagascalia 14(1): 25-34.

Giráldez Fernández, X. 1987. Algunos táxones interesantes del sureste zamorano. Collect. Bot. (Barcelona) 17: 107-111.

Ladero Álvarez, M.; NavarRo Andrés, F.; Valle GutiérReZ, C. J.; Marcos Laso, B.; Ruiz Téllez, T. \& Santos BobiLLO, M. T. 1984. Vegetación de los saladares castellano-leoneses. Stud. Bot. Univ. Salamanca 3: 17-62.

Llamas García, F. 1984. Flora y vegetación de la Maragatería (León). Inst. Fray Bernardino de Sahagún, Excma. Dip. Prov. León-CSIC, León.

LOSA EspaÑa, T. M. 1949. Contribución al estudio de la flora de la provincia de Zamora. Plantas de Sierra Segundera, La Puebla de Sanabria, Calabor, etc. Inst. A. J. Cavanilles, secc. Barcelona. CSIC, Barcelona.

LuCEÑo, M. \& Escudero, M. 2008. Carex L. En: S. Castroviejo, M. Luceño, A. Galán, P. Jiménez Mejías, F. Cabezas \& L. Medina (Eds), Flora iberica 18: 109-250. Real Jardín Botánico de Madrid, CSIC.

Mayor López, M.; Alonso Fernández, J. R.; Fernández CaSADO, M. A.; GutiérReZ, M. I.; HomeT, J.; NAVA, S. \& LASTRA MENÉnDeZ, J. J. 1983. Especies espinosas de los géneros Genista L. y Echinospartum (Spach.) Rothm. en el noroeste de la Península Ibérica. Bol. Inst. Estud. Asturianos, Supl. Ci. 31: 49-67.

MontserRat ReCoder, P. 1967. Florística ibérica. I. Bol. Real Soc. Esp. Hist. Nat., Secc. Biol. 65(1-2): 111-143.

Morales, R. 1998. Spiraea L. En: F. Muñoz Garmendia \& C. Navarro (Eds.), Flora iberica 6: 6-9. Real Jardín Botánico, CSIC, Madrid.
Nieto Feliner, G. 1985. Estudio crítico de la flora orófila del suroeste de León: Montes Aquilianos, Sierra del Teleno y Sierra de la Cabrera. Ruizia 2: 1-239.

Ramos NúÑEz, Á. F. 1993. Hypericum L. En: S. Castroviejo, C. Aedo, S. Cirujano, M. Laínz, P. Montserrat, R. Morales, F. Muñoz Garmendia, C. Navarro, J. Paiva \& C. Soriano (Eds.), Flora iberica 3: 157-185. Real Jardín Botánico, CSIC, Madrid.

Romo, A. M. 1983. El gènere Spiraea a la Península Ibérica. Collect. Bot. (Barcelona) 14: 537-541.

RozeIRA, A. 1944. A flora da província de Trás-Os-Montes e Alto Douro. Mem. Soc. Brot. 3: 114-117.

SÁnchez Anta, M. A. \& NavarRo Andrés, F. 1985. Peculiaridades cauloanatómicas adaptativas de algunas citiseas y genisteas orófilas. Lazaroa 8: 315-322.

SÁncheZ RodrígueZ, J. A. 1986. Aportaciones a la flora zamorana, I. Collect. Bot. (Barcelona) 16: 371-376.

SÁnchez Rodríguez, J. A. 1983. Flora y vegetación vascular de la comarca de Sayago (Zamora). Tesis doctoral, Universidad de Salamanca (inédita).

SesMA, J. \& LoIDI, J. 1992. Aportación 26, Spiraea hypericifolia L. subsp. obovata (Waldst. et Kit. ex Willd.) H. Huber. En: A. Molina \& A. Rubio (Eds.), Cartografía Corológica Ibérica. Bot. Complut. 17: 148-153.

Talavera, S. 2000. Genista L. En: S. Talavera, C. Aedo, S. Castroviejo, C. Romero Zarco, L. Saez, F. J. Salgueiro \& M. Velayos (Eds.), Flora iberica 7(2): 45-119. Real Jardín Botánico, CSIC, Madrid.

VALLE GuTIÉRREZ, C. J. 1982. Flora y vegetación vascular de las comarcas zamoranas de Tabara, Alba y Aliste. Tesis doctoral, Universidad de Salamanca (inédita).

Valle GuTIÉRReZ, C. J. 1984 Datos corológicos sobre plantas vasculares del centro-occidente zamorano. Stud. Bot. Univ. Salamanca 3: 295-299.

VARgas, P. 1997. Saxifraga L. En: S. Castroviejo, C. Aedo, M. Laínz, R. Morales, F. Muñoz Garmendia, G. Nieto Feliner \& J. Paiva (Eds.), Flora iberica 5: 162-242. Real Jardín Botánico, CSIC, Madrid.

VillaR, L. 1993. Vaccinium L. En: S. Castroviejo, C. Aedo, S. Cirujano, M. Laínz, P. Montserrat, R. Morales, F. Muñoz Garmendia, C. Navarro, J. Paiva \& C. Soriano (Eds.), Flora iberica 3: 519-523. Real Jardín Botánico, CSIC, Madrid. 